\title{
MODERN RESEARCH IN HIGH RESOLUTION INFRARED SPECTROSCOPY AND ITS IMPORTANCE \\ TO PLANETARY PHYSICS
}

\author{
K. NARAHARI RAO \\ Department of Physics, The Ohio State University, Columbus, Ohio, U.S.A.
}

\begin{abstract}
The scope of research in the field of high resolution infrared spectroscopy has expanded in numerous directions in recent years. Investigations of high resolution molecular spectra performed under controlled conditions in the laboratory are of significance to planetary studies because of the high resolution which one can achieve at the present time in the spectra of planets. In making use of laboratory data it is important to develop a proper perspective; due to technological advances the quality of experimental results has improved continuously during the past two decades, and, therefore, one has to exercise proper judgement when combining information from different laboratories. Somewhat detailed discussions are presented for the spectra of $\mathrm{CO}$ and $\mathrm{CO}_{2}$ to illustrate what modern measurements are capable of accomplishing. In the case of $\mathrm{CO}_{2}$, the extensive measurements of Courtoy and Herzberg done in the early 50's are not summarized in this paper because they are all consolidated in three previous publications. On the other hand, more recent measurements on $\mathrm{CO}_{2}$ are scattered in several publications and, therefore, an attempt has been made to collect them together in this article. This is not a comprehensive review paper but it is intended to provide only a glimpse into the expanding frontiers of research in high resolution infrared spectroscopy.
\end{abstract}

\section{Introduction}

During the past decade many notable advances have been made in the field of high resolution infrared spectroscopy as a result of which the scope and applicability of the field has expanded by leaps and bounds.

From a spectroscopist's point of view, by studying high resolution infrared spectra of molecules we are primarily contributing to the understanding of the structures of molecules in their ground electronic states. By this we mean, determination of the geometrical arrangement of the nuclei in molecules, obtaining precise values for internuclear distances and bond angles and evaluation of force fields applicable to molecular vibrations. For achieving these goals, it is necessary to obtain precision in spectral positions and intensities and develop procedures for correlating experimental results with theory.

The sophisticated technology (Rao and Mathews, 1972) that has become available to us in recent years has been enabling us not only to achieve the above objectives better than ever before but has contributed to the expansion of the capabilities of the field in numerous other directions. This is illustrated in the following summary which gives an overall view of many of the current interests in high resolution infrared spectroscopy.

Structures of Molecules: $\left\{\begin{array}{l}\text { Molecular Geometry } \\ \text { Internuclear Distances } \\ \text { Force Fields }\end{array}\right.$

Planetary and Stellar Spectra: Fourier Spectroscopy

(since 1962) 
Molecular Laser Emissions:

Transitions between levels not attained ordinarily by absorption spectroscopy Earth's Atmosphere:

Photochemistry of stratosphere (in particular)

$\mathrm{NO}_{2}, \mathrm{HNO}_{3}$, (identified 1968)

Concentrations of $\mathrm{O}_{3}, \mathrm{H}_{2} \mathrm{O}, \mathrm{NO}_{2}, \mathrm{CH}_{4}, \mathrm{~N}_{2} \mathrm{O}, \mathrm{HNO}_{3}, \mathrm{CO}, \mathrm{NO} \ldots$ (requiring measurement)

First, the high resolution attained in the infrared spectra of cosmic sources by employing techniques in Fourier transform spectroscopy has provided a new dimension to the type of information available to astrophysicists. Second, the molecular laser emissions are playing a significant role in spectroscopic research because a large number of the energy levels involved in the transitions of the laser lines are unusual in that they are not easily reached by conventional absorption and emission techniques in infrared spectroscopy. Finally, in view of future large scale supersonic transport operations, the need to understand more about our own stratosphere has become important and for this purpose the experimental studies of stratospheric constituents conducted in the laboratory under controlled conditions have assumed vital significance.

There is much unity in all these aspects of research in high resolution infrared spectroscopy and a few examples will be discussed in this paper in order to emphasize that scientists involved in any one of these areas should develop more than a passing interest in all the other areas.

\section{Improved Planetary Spectra in the Infrared}

Use of Fourier transform spectroscopy (FTS) in the recording of planetary spectra in the infrared has provided a gain in the resolving power in the spectrum of Venus by a factor of at least 100 as compared to the best spectra recorded with a scanning grating spectrometer. In addition to high resolution capabilities, the techniques in Fourier spectroscopy enable the determination of spectral positions with high accuracy and, therefore, comparison of planetary data with laboratory measurements is easier to make. As a result of such superior quality data obtained for planetary spectra, Connes and associates made important discoveries. For instance, they were able to identify for the first time the presence of $\mathrm{CO}, \mathrm{HCl}$ and $\mathrm{HF}$ in the Venus atmosphere (Connes et al., 1967, 1968) and of CO in Mars (Kaplan et al., 1969).

\section{Large Gratings and Fourier Transform Spectrometers}

By high resolution infrared spectra we refer to the ones in which the measured full widths at half heights in unblended spectral lines are at least of the order of $0.02 \mathrm{~cm}^{-1}$. At the present time, it is possible to achieve this kind of spectral resolution in day-today observations obtained with spectrographs equipped with large modern plane 
gratings (with dimensions $40 \mathrm{~cm} \times 20 \mathrm{~cm}$ ) (Rao and Mathews, 1972). In the context of the present paper, spectrographs equipped with such large gratings and Fourier transform spectrometers are considered to be the two kinds of instruments which are important for obtaining good high resolution infrared spectra. These instruments have complementary advantages. If employed properly, Fourier transform spectroscopy leads to high spectral resolution and with it one can determine wavelengths relative to the primary standard of length. Moreover, the use of high quality gratings for recording spectra is a well-established and well-understood technique; not only is it possible to achieve precision in the determination of spectral positions with grating instruments but it is very necessary to use them in research work involving the determination of intensities of spectral lines. This is because we want to assure ourselves that information is not lost as might happen in the mathematical operations used in obtaining Fourier transforms.

At the present time, for specific investigations, tunable lasers are helpful in attaining very high resolving power and, with this technique there will undoubtedly be attempts made to achieve accuracy in spectral positions as well as ability to scan over large extents of the infrared spectrum. To be sure, the vibration rotation bands of molecules occur in different parts of the near infrared between 1-30 $\mu$, and for many spectroscopic studies it is important to have available as much information as feasible for as many bands as possible.

\section{Aspects of the Infrared Spectrum of CO}

The infrared spectrum of the $\mathrm{CO}$ molecule continues to provide fascinating possibilities for research. The interdisciplinary nature of infrared spectroscopic research can be recognized when we consider a few of the numerous types of programs undertaken involving the spectra of this molecule. Many of these programs are the result of recent research. Mention has already been made of the occurrence of $\mathrm{CO}$ in the atmospheres of Venus and Mars. In 1970, the CO molecule was identified (Wilson et al., 1970) as one of the more abundant molecules in interstellar space. In the laboratory measurement of high resolution infrared spectra, the infrared $\mathrm{CO}$ lines have been useful as secondary standards of wavelength (Rao, 1950). It is well known that studies of the abundance of this molecular species in the Earth's atmosphere have been (Migeotte, 1959; Migeotte and Neven, 1950; Locke and Herzberg, 1953) and continue to be of interest to atmospheric scientists. Finally, studies of CO laser spectra (Mantz et al., 1970) have enabled a critical examination (Mantz et al., 1971 ; Fleming and Rao, 1972; Kirschner and Watson, 1973) of the methods of developing the potential functions of diatomic molecules. This last aspect will be amplified a little more because of its impact in interpreting recent observations of solar spectra.

First, a few comments about the absorption spectrum of $\mathrm{CO}$ in the infrared: The vibration-rotation fundamental of $\mathrm{CO}$ at $4.7 \mu$ is easy to study by absorption techniques. It requires only an absorption path of about $2 \mathrm{~cm}$-atm to observe. The spectrum presents a simple appearance and the spacings between the successive lines in its 
rotational structure $\left(3-4 \mathrm{~cm}^{-1}\right)$ is such that, as mentioned earlier, $\mathrm{CO}$ lines have been useful in calibrating infrared spectra. The third overtone band of $\mathrm{CO}$ (4-0 band) occurs in the photographic infrared and Herzberg and Rao (1949) used an absorption path of nearly 800 meters at a pressure of 1 atm to observe it.

Figure 1 shows a sketch of the potential energy curve for $\mathrm{CO}$. It may be noted that absorption studies as indicated above allow us to obtain information for only a very small portion of the potential energy curve. Attempts to evaluate the potential energy function for the ground electronic state of $\mathrm{CO}$ have met with some measure of success only during recent years. The energy levels sketched in Figure 1 up to $v=37$ can all be studied experimentally from studies of the infrared spectra of a CO laser (Roh and Rao, 1974). The vibrational and rotational term value expression used to fit the observational data including all the laser transitions is given below:

$$
\begin{aligned}
T(v, J)= & G(v)+F_{v}(J) \\
= & \omega_{e}\left(v+\frac{1}{2}\right)-\omega_{e} x_{e}\left(v+\frac{1}{2}\right)^{2}+\omega_{e} y_{e}\left(v+\frac{1}{2}\right)^{3}-\omega_{e} z_{e}\left(v+\frac{1}{2}\right)^{4}+ \\
& +\omega_{e} a_{e}\left(v+\frac{1}{2}\right)^{5}-\omega_{e} b_{e}\left(v+\frac{1}{2}\right)^{6}+\cdots+ \\
& +J(J+1)\left[B_{e}-\alpha_{e}\left(v+\frac{1}{2}\right)+\gamma_{e}\left(v+\frac{1}{2}\right)^{2}-\cdots\right]- \\
& -J^{2}(J+1)^{2}\left[D_{e}-\beta_{e}\left(v+\frac{1}{2}\right)+\varepsilon_{e}\left(v+\frac{1}{2}\right)^{2}-\cdots\right]+ \\
& +J^{3}(J+1)^{3}\left[H_{e}-\delta_{e}\left(v+\frac{1}{2}\right)+\cdots\right]-\cdots .
\end{aligned}
$$

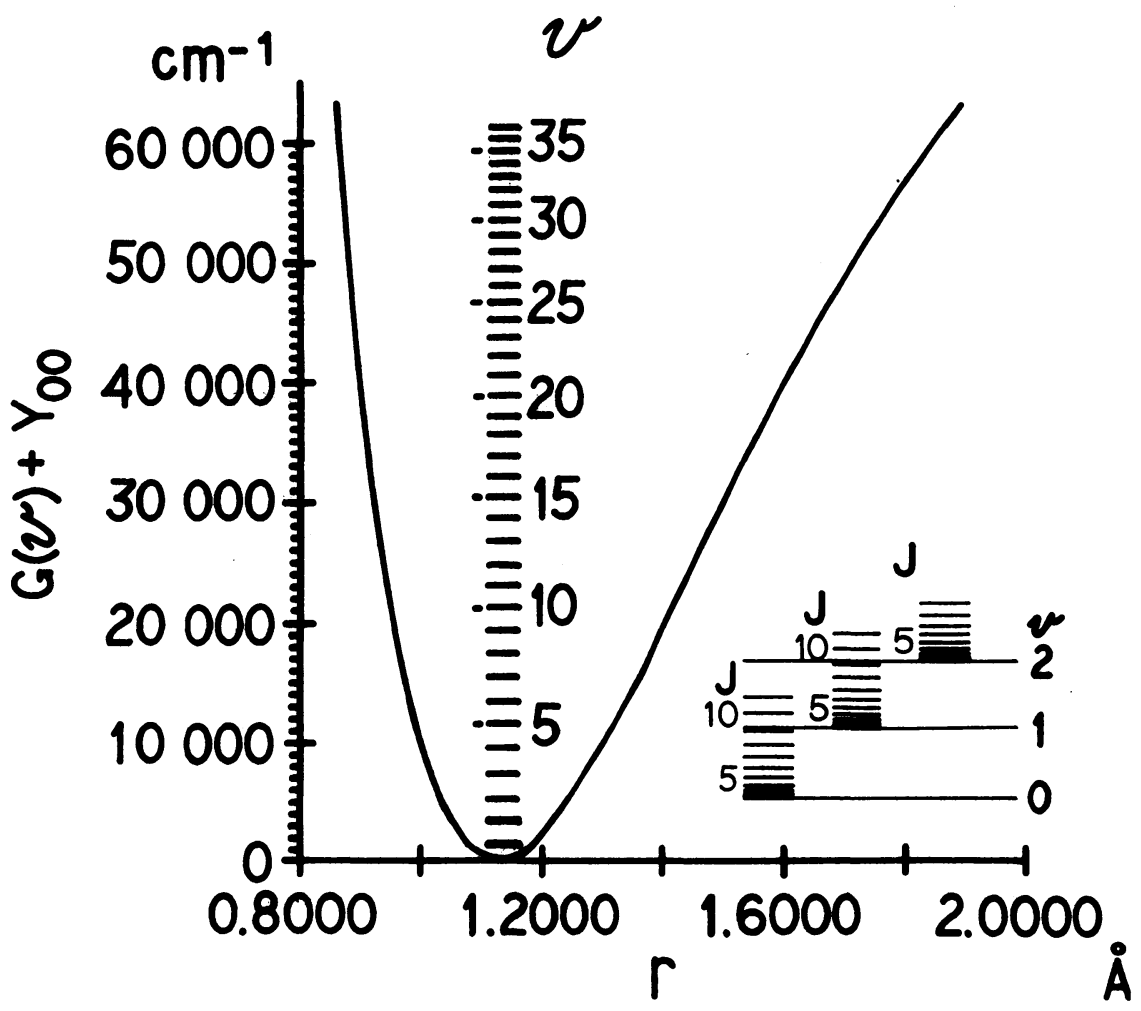

Fig. 1. Vibrational energy levels observed in a $\mathrm{CO}$ laser. 
The numerical values for the constants obtained from experimental data for ${ }^{12} \mathrm{C}^{16} \mathrm{O}$ are presented in Equation (2) expressed in $\mathrm{cm}^{-1}$.

$$
\begin{aligned}
T(v, J)= & (2169.817346 \pm 0.000670)\left(v+\frac{1}{2}\right)- \\
& -(13.289545 \pm 0.000280)\left(v+\frac{1}{2}\right)^{2}+ \\
& +\left(1.06521 \times 10^{-2} \pm 0.00350 \times 10^{-2}\left(v+\frac{1}{2}\right)^{3}+\right. \\
& +\left(5.037 \times 10^{-5} \pm 0.197 \times 10^{-5}\right)\left(v+\frac{1}{2}\right)^{4}+ \\
& +\left(1.1486 \times 10^{-6} \pm 0.0510 \times 10^{-6}\right)\left(v+\frac{1}{2}\right)^{5}- \\
& -\left(3.3124 \times 10^{-8} \pm 0.0484 \times 10^{-8}\right)\left(v+\frac{1}{2}\right)^{6}+\cdots+ \\
& +J(J+1)\left[\left(1.931283656 \pm 484 \times 10^{-9}\right)-\right. \\
& -\left(1.751003 \times 10^{-2} \pm 0.000110 \times 10^{-2}\right)\left(v+\frac{1}{2}\right)+ \\
& \left.+\left(1.7214 \times 10^{-6} \pm 0.0260 \times 10^{-6}\right)\left(v+\frac{1}{2}\right)^{2}-\cdots\right]- \\
& -J^{2}(J+1)^{2}\left[\left(6.125781 \times 10^{-6} \pm 0.000780 \times 10^{-6}\right)-\right. \\
& -\left(2.927 \times 10^{-9} \pm 0.437 \times 10^{-9}\right)\left(v+\frac{1}{2}\right)+ \\
& \left.+\left(4.313 \times 10^{-10} \pm 0.763 \times 10^{-10}\right)\left(v+\frac{1}{2}\right)^{2}-\cdots\right]+ \\
& +J^{3}(J+1)^{3}\left[\left(7.723 \times 10^{-12} \pm 0.749 \times 10^{-12}\right)-\right. \\
& \left.-\left(3.364 \times 10^{-13} \pm 0.772 \times 10^{-13}\right)\left(v+\frac{1}{2}\right)+\cdots\right]+\cdots
\end{aligned}
$$

The error limits quoted in the above equation for each constant are the standard deviations. In evaluating these molecular constants, by least squares techniques, other measurements in the infrared (Rao et al., 1966; Rank et al., 1957, 1965; Weinberg et al., 1965) and in the microwave regions (Rosenbloom et al., 1958; Cowan and Gordy, 1957; Helminger et al., 1970) have been included by weighting the data proportional to $(\Delta v)^{-2}$, where $\Delta v$ is the estimated uncertainty of measurements.

Now, we come to the importance of all this work to astrophysics. In the June 1973 issue of the Astrophysical Journal, Hall (1973) reported some solar data in the fundamental region of the $\mathrm{CO}$ molecule at $4.7 \mu$ in the infrared. This is a continuation of his work in the first overtone region at $2.3 \mu$. He has a much richer $\mathrm{CO}$ spectrum at $4.7 \mu$ and in the ordinary variety of carbon monoxide (viz., ${ }^{12} \mathrm{C}^{16} \mathrm{O}$ ) he reported transitions involving high $J(J>100)$ between vibrational levels up to $v=7$. He also announced the detection of the isotopic varieties ${ }^{13} \mathrm{C}^{16} \mathrm{O},{ }^{12} \mathrm{C}^{18} \mathrm{O}$ and ${ }^{12} \mathrm{C}^{17} \mathrm{O}$ in the solar spectra. These identifications have been made by calculating the $\mathrm{CO}$ spectrum employing the molecular constants involving laser data up to $v=28$ (Mantz et al., 1970). For evaluating the spectra of the isotopic varieties, Hall employed the theoretical relations given by Dunham (1932). All these CO studies demonstrate the aspect of relationship between the different areas of interest in high resolution infrared spectroscopy mentioned earlier in this paper. Incidentally, the results of Hall are consistent with the hypothesis that in the solar atmosphere all the three isotopes, viz., carbon-13, oxygen-18 and oxygen-17 are within their terrestrial abundances.

Before closing the discussion of the $\mathrm{CO}$ molecule, it should be mentioned that the solar data were not included in the least squares program mentioned above for determining molecular constants. The constants are sensitive to the weighting of the observational data which, as stated earlier, is proportional to $(\Delta v)^{-2}$. There was no clear indication about the observational uncertainties $(\Delta v)$ of the solar data. When that 
aspect is clarified it would indeed be highly desirable to include the solar data for the types of computations of interest to molecular spectroscopists. In fact, as may be seen below, the values calculated for some of the solar lines from Equation (2) do not quite agree with the measurements (Hall, 1973).

\section{TABLE I}

Wave numbers (expressed in vac. $\mathrm{cm}^{-1}$ ) for the 7-6 band of ${ }^{12} \mathrm{C}^{16} \mathrm{O}$

\begin{tabular}{rlll}
\hline$R(J)$ & Solar data & Calc. $^{\mathrm{a}}$ & Dif. \\
\hline$R 65$ & 2140.930 & 2140.913 & 0.017 \\
66 & & & \\
67 & 2142.811 & 2142.796 & 0.015 \\
68 & 2143.688 & 2143.669 & 0.019 \\
69 & & & \\
70 & & & \\
71 & 2146.056 & 2146.018 & 0.038 \\
$R 101$ & 2146.361 & 2146.119 & 0.242 \\
102 & & & \\
103 & 2144.929 & 2144.552 & 0.377 \\
104 & 2144.156 & 2143.692 & 0.464 \\
105 & 2143.336 & 2142.781 & 0.555 \\
106 & & & \\
107 & & & \\
108 & 2140.603 & 2139.738 & 0.865 \\
\hline
\end{tabular}

a The author wishes to thank Dr Won B. Roh for supplying this information.

This suggests two possibilities: a more careful study of the solar spectrum should be made especially because the (7-6) band forms a head and the lines $R(101)-R(108)$ form the returning branch. On the other hand, it is entirely possible that further refinements should be made in the $\mathrm{CO}$ constants by including more experimental data especially those involving high $J$ values.

\section{5. $\mathrm{CO}_{2}$}

The infrared spectra of this molecule provide an example of how by combining the laboratory data with properly measured planetary infrared spectra it has been possible to obtain valuable information both for the structure of this molecule as well as for the understanding of the planetary atmospheres. Good high resolution data are available for several infrared bands of the ordinary variety of carbon dioxide, namely, ${ }^{12} \mathrm{C}^{16} \mathrm{O}_{2}$. With somewhat less precision are the ${ }^{13} \mathrm{C}^{16} \mathrm{O}_{2}$ bands known. In view of the importance of this molecule it would be useful to summarize the available data for these and other isotopic varieties of carbon dioxide and that has been done in Tables II-VIII. The ground state molecular constants available for the different isotopic species of carbon dioxide are given in Table IX. Combining the results obtained 
TABLE II

Molecular constants (expressed in $\mathrm{cm}^{-1}$ ) of ${ }^{12} \mathrm{C}^{16} \mathrm{O}_{2}$ bands

Reference numbers refer to the references typed as a footnote to this table and they are not the same as references in the text)

\begin{tabular}{|c|c|c|c|c|c|c|}
\hline Levels & & $v_{0}$ & $\left(B^{\prime}-B^{\prime \prime}\right) \times 10^{5}$ & $\left(D^{\prime}-D^{\prime \prime}\right) \times 10^{8}$ & Ref. & Other references \\
\hline Upper & Lower & & & & & \\
\hline $03^{10}$ & $10^{0} 0$ & 544.26 & 58 & 2.75 & 3 & \\
\hline $\begin{array}{l}03^{1 c} 0 \\
03^{1 d} 0\end{array}$ & $\begin{array}{l}02^{2 c} 0 \\
02^{2 d} 0\end{array}$ & $597.337 \pm 0.008$ & $\begin{array}{r}-90.1 \\
1.8\end{array}$ & & 8 & 3,7 \\
\hline $02^{\circ} 0$ & $01^{1 c} 0$ & $618.033 \pm 0.005$ & -15.9 & 2.3 & 8 & $3,7,21$ \\
\hline $\begin{array}{l}01^{1 c 0} \\
01^{1 d} 0\end{array}$ & $\begin{array}{l}00^{9} 0 \\
00^{\circ} 0\end{array}$ & $667.380 \pm 0.005$ & $\begin{array}{r}42.4 \pm 0.7 \\
103.7 \pm 0.9\end{array}$ & $\begin{array}{l}0.3 \pm 0.3 \\
0.3 \pm 0.3\end{array}$ & 19 & $2,3,5,7,8,20,21$ \\
\hline $02^{2 c} 0$ & $01^{1 c} 0$ & & & 0.1 & & \\
\hline $02^{2 a} 0$ & $01^{1 d} 0$ & $667.750 \pm 0.005$ & 41.2 & 0.0 & 8 & 3,7 \\
\hline $10^{0} 0$ & $01^{1 c} 0$ & $720.808 \pm 0.005$ & -43.4 & -1.0 & 8 & $3,7,21$ \\
\hline $11^{1 c} 0$ & $\begin{array}{l}02^{2 c} 0 \\
02^{2} a_{0}\end{array}$ & $741.730 \pm 0.008$ & $\begin{array}{l}-128.5 \\
-\quad 321\end{array}$ & & 8 & 3,7 \\
\hline $11^{1 d} 0$ & $\begin{array}{l}02^{2 \omega_{0}} 0 \\
03^{3} 0\end{array}$ & 757.47 & $\begin{array}{l}-32.1 \\
-84\end{array}$ & -0.8 & 3 & \\
\hline $\begin{array}{l}12^{2} 0 \\
11^{10}\end{array}$ & $\begin{array}{l}03^{3} 0 \\
02^{0} 0\end{array}$ & 791.43 & $\begin{array}{r}-7 \\
-\quad 7\end{array}$ & -2.75 & 3 & \\
\hline $01^{1 c} 1$ & $11^{1 c} 0$ & 927.17 & -303 & & 12 & 11 \\
\hline $01^{1 d} 1$ & $\begin{array}{l}11^{1 d} 0 \\
10^{0} 0\end{array}$ & 960.95857 & -304.739 & 1.837 & 1 & 4.15 \\
\hline $00^{0} 1$ & $\begin{array}{l}10^{\circ} 0 \\
02^{\circ} 0\end{array}$ & $\begin{array}{r}960.95857 \\
1063.73457\end{array}$ & -334.076 & -2.382 & 1 & $\begin{array}{l}4,15 \\
4,15\end{array}$ \\
\hline $00^{0} 1$ & $\begin{array}{l}02^{\circ} 0 \\
00^{\circ} 0\end{array}$ & $\begin{array}{l}1063.73457 \\
1285.40\end{array}$ & 19 & & 22 & \\
\hline $02^{\circ} 0$ & $\begin{array}{l}00^{\circ} 0 \\
00^{\circ} 0\end{array}$ & $\begin{array}{l}1285.40 \\
1388.15\end{array}$ & -3 & & 22 & \\
\hline $10^{\circ} 0$ & $\begin{array}{l}00^{\circ} 0 \\
00^{\circ} 0\end{array}$ & & $51.3 \pm 0.2$ & $1.1 \pm 0.2$ & & \\
\hline $\begin{array}{l}03^{1 c 0} \\
03^{1 d} 0\end{array}$ & $\begin{array}{l}00^{\circ} 0 \\
00^{9} 0\end{array}$ & $1932.477 \pm 0.004$ & $146.7 \pm 0.4$ & & 13 & 3,7 \\
\hline $11^{1 c 0}$ & $00^{9} 0$ & & $16.7 \pm 0.2$ & $-0.7+0.1$ & & \\
\hline $11^{11} 0$ & $00^{\circ} 0$ & $20 / 6.890 \pm 0.003$ & $110.1 \pm 0.3$ & & 13 & 3 \\
\hline $12^{2 c 0}$ & $\begin{array}{l}01^{1 c 0} \\
01^{1 d} d_{0}\end{array}$ & $2093.356 \pm 0.004$ & $89.2 \pm 0.9$ & & 13 & 3 \\
\hline $12^{2 d} 0$ & $\begin{array}{l}01^{1 d} 0 \\
01^{1 c} 0\end{array}$ & $2129.769+0.005$ & $\begin{array}{r}28.2+0.9 \\
-\quad 3.3+0.5\end{array}$ & & 13 & 3 \\
\hline $20^{\circ} 0$ & $\begin{array}{l}01^{1 c} 0 \\
00^{0} 8\end{array}$ & $2150.65 \pm 0.02$ & $\begin{array}{l}-3.3 \pm 0.5 \\
-304+7\end{array}$ & $1+6$ & 19 & \\
\hline 0009 & $\begin{array}{l}00^{0} 8 \\
00^{07}\end{array}$ & $2175.268 \pm 0.007$ & $-305.1 \pm 1.8$ & $0.4+0.9$ & 19 & \\
\hline 0008 & $\begin{array}{l}00^{07} \\
00^{0} 6\end{array}$ & $2199.953 \pm 0.005$ & $-306.6+1.5$ & $-0.4+0.8$ & 19 & \\
\hline 0007 & $\begin{array}{l}00^{0} 6 \\
00^{0} 5\end{array}$ & $2224.697 \pm 0.004$ & $-307.2 \pm 1.3$ & $-0.42 \pm 0.49$ & 19 & \\
\hline $00^{0} 6$ & $\begin{array}{l}00^{05} \\
00^{0} 4\end{array}$ & $2249.492 \pm 0.003$ & $-305.6 \pm 0.6$ & $0.34 \pm 0.31$ & 19 & \\
\hline $\begin{array}{l}00^{05} \\
00^{0} 4\end{array}$ & $\begin{array}{l}00^{0} 4 \\
00^{03}\end{array}$ & $2274.355 \pm 0.002$ & $-307.3 \pm 0.4$ & $-0.19 \pm 0.15$ & 19 & \\
\hline $00^{03}$ & $00^{0} 2$ & $2299.255 \pm 0.001$ & $-307.35 \pm 0.19$ & $0.00 \pm 0.08$ & 19 & 10 \\
\hline $02^{2 c 1}$ & $02^{2 c} 0$ & $2324142+0005$ & $-302.3 \pm 1.0$ & $0.6 \pm 0.6$ & 16 & 10 \\
\hline $02^{2 d} 1$ & $02^{2 d} 0$ & $2324.142 \pm 0.005$ & $-302.3 \pm 1.0$ & $0.6 \pm 0.6$ & & \\
\hline $00^{0} 2$ & $00^{0} 1$ & $2324.188 \pm 0.001$ & $-307.67 \pm 0.15$ & $-0.04 \pm 0.06$ & 19 & 10 \\
\hline $10^{0} 1$ & $10^{\circ} 0$ & $2326.600 \pm 0.005$ & $-312.8 \pm 1.0$ & & 16 & 10 \\
\hline $02^{0} 1$ & $02^{0} 0$ & $2327.433 \pm 0.005$ & $-297.9 \pm 1.0$ & $0.08 \pm 0.1$ & 10 & \\
\hline $01^{1 c 1}$ & $\begin{array}{l}01^{1 \mathrm{c}} 0 \\
01^{1 d} 0\end{array}$ & $2236.633 \pm 0.003$ & $-304.4 \pm 0.5$ & $0.00 \pm 0.1$ & 16 & 10 \\
\hline $\begin{array}{l}01^{1 d} 1 \\
00^{0} 1\end{array}$ & $\begin{array}{l}01^{1 d} 0 \\
00^{0} 0\end{array}$ & $2349.147 \pm 0.001$ & $\begin{array}{l}-306.4 \pm 0.5 \\
-307.88 \pm 0.07\end{array}$ & $\begin{aligned} 0.00 & \pm 0.1 \\
-0.05 & \pm 0.02\end{aligned}$ & 19 & $9,10,14,15,16,17$ \\
\hline $10^{\circ} 1$ & $02^{0} 0$ & 2429.37 & -341 & -3.5 & 15 & \\
\hline $01^{1 c 1}$ & $00^{\circ} 0$ & $3004.016 \pm 0.005$ & $-262.3 \pm 0.7$ & $-0.3 \pm 0.3$ & 19 & \\
\hline $01^{1 d} 1$ & $00^{\circ} 0$ & $500+.010 \pm 0.005$ & $-202.9 \pm 0.9$ & $-0.3+0.3$ & 19 & \\
\hline $05^{1} 0$ & $00^{\circ} 0$ & 3181.45 & 80 & & 18 & \\
\hline $13^{10}$ & $00^{\circ} 0$ & 3339.34 & -19 & 0.0 & 18 & \\
\hline $05^{3} 1$ & $03^{3} 0$ & 3528.25 & -277 & & 18 & \\
\hline
\end{tabular}


Table II (Continued)

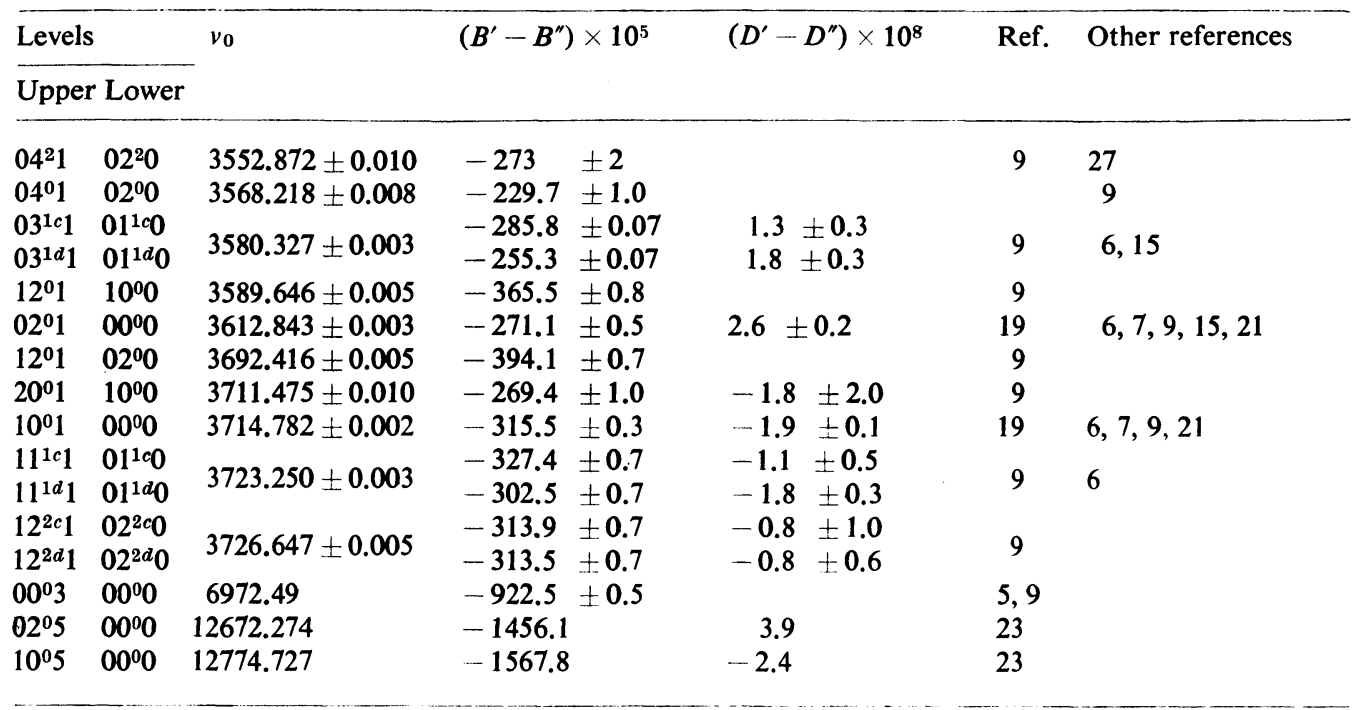

\section{Important Note}

In addition to the above data, $v_{0}, \Delta B$ and $\Delta D$ values for several other bands of ${ }^{12} \mathrm{CO}_{2}$ can be obtained from the following three classic publications. The constants quoted in these papers have been obtained by graphical techniques. Since the basic measurements are also given in these papers they can be reprocessed should that become necessary.

I. G. Herzberg and L. Herzberg, 'Rotation-Vibration Spectra of Diatomic and Simple Polyatomic Molecules with Long Absorbing Paths. XI: The Spectrum of Carbon Dioxide $\left(\mathrm{CO}_{2}\right)$ Below 1.25 $\mu$ ', J. Opt. Soc. Amer. 43, 1037 (1953).

II. C. P. Courtoy, 'Spectres de vibration-rotation de molécules simples diatomiques ou polyatomiques avec long parcours d'absorption. XII: Le spectre de ${ }^{12} \mathrm{C}^{16} \mathrm{O}_{2}$ entre 3500 et $8000 \mathrm{~cm}^{-1}$ et les constantes moléculaires de cette molécule', Can. J. Phys. 35, 608 (1957).

III. C. P. Courtoy, 'Spectre infrarouge à grande dispersion et constantes moléculaires du $\mathrm{CO}_{2}$ ', Ann. Soc. Sci. Bruxelles, Sér I: Math. Astron. Phys. 73, 5 (1959).

References for ${ }^{12} \mathrm{C}^{16} \mathrm{O}_{2}$ data

1. K. M. Baird, H. D. Riccius, and K. J. Siemson, Opt. Commun. 6, 91 (1972); see also T. Y. Chang, Opt. Commun. 2, 77 (1970).

2. Joseph J. Barrett and Alfons Weber, J. Opt. Soc. Am. 60, 70 (1970).

3. W. S. Benedict, Mem. Soc. Roy. Sci. Liège 2, 18 (1957). [M. Migeotte, L. Neven, and J. Swensson, 'The Solar Spectrum from 2.8 to 23.7 Microns. II: Measures and Identifications.]

4. T. J. Bridges and T. Y. Chang, Phys. Rev. Letters 22, 811 (1969).

5. Charles P. Courtoy, Ann. Soc. Sci. Bruxelles, Ser. 1: Math. Astron. Phys. 73, 5 (1959).

6. W. L. France and F. P. Dickey, J. Chem. Phys. 23, 471 (1955).

7. Howard R. Gordon, Ph. D. Dissertation, The Pennsylvania State University (1965).

8. Howard R. Gordon and T. K. McCubbin, Jr., J. Mol. Spectrosc. 18, 73 (1965).

9. Howard R. Gordon and T. K. McCubbin, Jr., J. Mol. Spectrosc. 19, 137 (1966).

10. Yu Hak Hahn, Ph. D. Dissertation, The Pennsylvania State University (1967).

11. B. Hartmann and B. Kleman, Can. J. Phys. 44, 1609 (1966).

12. John A. Howe and R. A. McFarlane, J. Mol. Spectrosc. 19, 224 (1966).

13. Arthur G. Maki, Earle K. Plyler, and Robert J. Thibault, J. Res. Nat. Bur. Stand. 67A, 219 (1963)

14. T. K. McCubbin, Jr., and Yu Hak Hahn, J. Opt. Soc. Am. 57, 1373 (1967).

15. M. Migeotte, L. Neven, and J. Swensson, Mem. Soc. Roy. Sci. Liège 2, 5 (1957).

16. Ralph Oberly, K. Narahari Rao, Y. H. Hahn, and T. K. McCubbin, Jr., J. Mol. Spectrosc. 25, 138 (1968). 
17. Earle K. Plyler, Lamdin R. Blaine, and Eugene D. Tidwell, J. Res. Nat. Bur. Stand. 55, 183 (1955).

18. Earle K. Plyler, Eugene D. Tidwell, and W. S. Benedict, J. Opt. Soc. Am. 52, 1017 (1962).

19. Robert Earl Pulfrey, Ph. D. Dissertation, The Pennsylvania State University (1972).

20. Kurt Rossmann, K. Narahari Rao, and Harald H. Nielsen, J. Chem. Phys. 24, 103 (1956).

21. Kurt Rossmann, W. L. France, K. Narahari Rao, and H. H. Nielsen, J. Chem. Phys. 24, 1007 (1956).

22. B. P. Stoicheff, Can. J. Phys. 36, 218 (1958).

23. L. D. Gray Young, A. T. Young, and R. A. Schorn, J. Quant. Spectrosc. Radiat. Transfer 10, 1291 (1970).

TABLE III

Molecular constants (expressed in $\mathrm{cm}^{-1}$ ) of ${ }^{13} \mathrm{C}^{16} \mathrm{O}_{2}$

\begin{tabular}{|c|c|c|c|c|}
\hline \multicolumn{2}{|l|}{ Levels } & \multirow[t]{2}{*}{$v_{0}$} & \multirow[t]{2}{*}{$\left(B^{\prime}-B^{\prime \prime}\right) \times 10^{5}$} & \multirow[t]{2}{*}{$\left(D^{\prime}-D^{\prime \prime}\right) \times 10^{8}$} \\
\hline Upper & Lower & & & \\
\hline $11^{1 c} 0$ & $00^{3} 0^{\mathrm{a}}$ & $2037.093 \pm 0.006$ & $-210.0 \pm 2.0$ & $1.40 \pm 0.70$ \\
\hline $01^{1 c} 1$ & $01^{1 c} 0^{b}$ & & $-292.8 \pm 1.0$ & \\
\hline $01^{1 d} 1$ & $01^{1 d} 0^{b}$ & $2271.764 \pm 0.005$ & $-293.6 \pm 1.0$ & \\
\hline $00^{0} 1$ & $00^{0} 0^{\mathrm{b}}$ & $2283.490 \pm 0.004$ & $-296.3 \pm 1.0$ & \\
\hline $04^{2 c} 1$ & $02^{20} 0$ & & $-235.1 \pm 0.5$ & \\
\hline $04^{2 d_{1}}$ & $02^{2 d} 0$ & $3473.673 \pm 0.003$ & $-235.3 \pm 0.5$ & \\
\hline $04^{01}$ & $02^{\circ} 0$ & $3482.202 \pm 0.002$ & $-209.7 \pm 0.4$ & \\
\hline $03^{1 d} 1$ & $01^{1} 0$ & $3498.718 \pm 0.002$ & $-209.8 \pm 0.4$ & $1.96 \pm 0.14$ \\
\hline $12^{0} 1$ & $10^{00}$ & $3517.285 \pm 0.002$ & $-289.6 \pm 0.3$ & \\
\hline $02^{0} 1$ & $00^{\circ} 0$ & $3527.706 \pm 0.001$ & $-220.9 \pm 0.2$ & $2.20 \pm 0.03$ \\
\hline $20^{0} 1$ & $10^{\circ 0}$ & $3621.260 \pm 0.005$ & $-299.1 \pm 0.7$ & \\
\hline $12^{01}$ & $02^{00}$ & $3621.531 \pm 0.006$ & $-407.8 \pm 0.8$ & \\
\hline $10^{0} 1$ & $00^{0} 0^{c}$ & $3632.917 \pm 0.005$ & $-352.4 \pm 0.7$ & $-1.70 \pm 0.30$ \\
\hline $10^{0} 1$ & 0000 & $3632.879 \pm 0.002$ & $-351.5 \pm 0.3$ & $-1.46 \pm 0.08$ \\
\hline $11^{1 c 1}$ & $01^{1 c 0}$ & & $-345.8 \pm 0.1$ & \\
\hline $11^{1 d} 1$ & $01^{1 d} 0$ & $3639.182 \pm 0.2$ & $-327.8 \pm 0.1$ & \\
\hline $12^{2 c 1}$ & $02^{2 c 0}$ & & $-332.9 \pm 0.7$ & \\
\hline $12^{2 d} 1$ & $02^{2 d} 0$ & $3641.343 \pm 0.004$ & $-333.3 \pm 0.8$ & \\
\hline $06^{2} c_{1}$ & $02^{2 c 0}$ & & $-179.9 \pm 1.5$ & \\
\hline $06^{2 d} 1$ & $02^{2 d} 0$ & $4673.657 \pm$ & $-171.0 \pm 1.3$ & \\
\hline $06^{0} 1$ & $02^{00}$ & $4685.641 \pm 0.011$ & $-211.0 \pm 1.6$ & \\
\hline $05^{1 c} 1$ & $01^{100}$ & $4708475+03$ & $-199.1 \pm 0.6$ & $1.77 \pm 0.26$ \\
\hline $05^{1 d} 1$ & $01^{1 d} 0$ & $4 / 08.4 / 5 \pm 0.3$ & $-111.8 \pm 0.5$ & $4.54 \pm 0.21$ \\
\hline $04^{01}$ & $00^{\circ} 0$ & $4748.016 \pm 0.002$ & $-140.1 \pm 0.3$ & $4.68 \pm 0.08$ \\
\hline $14^{0} 1$ & $02^{00}$ & $4853.761 \pm 0.005$ & $-339.1 \pm 0.8$ & \\
\hline $14^{2} 1$ & $02^{2} 0$ & $4858.143 \pm 0.017$ & $-323.8 \pm 0.5$ & \\
\hline $13^{1 c 1}$ & $01^{100}$ & & $-352.3 \pm 0.1$ & \\
\hline $13^{1 d} 1$ & $01^{1 d} 0$ & $4871.403 \pm 0.002$ & $-302.2 \pm 0.1$ & \\
\hline $22^{\circ} 1$ & $10^{0} 0$ & $4871.857 \pm 0.004$ & $-384.9 \pm 0.6$ & \\
\hline $12^{01}$ & $00^{\circ} 0$ & $4887.343 \pm 0.002$ & $-338.6 \pm 0.2$ & $1.52 \pm 0.05$ \\
\hline $22^{0} 1$ & $02^{\circ} 0$ & $4976.085 \pm 0.009$ & $-499.9 \pm 2.2$ & \\
\hline $20^{\circ} 1$ & $00^{\circ} 0$ & $4991.304 \pm 0.002$ & $-352.9 \pm 0.3$ & $-3.79 \pm 0.06$ \\
\hline $30^{0} 1$ & $10^{\circ} 0$ & $4993.503 \pm 0.004$ & $-270.0 \pm 1.1$ & \\
\hline $21^{1 c} 1$ & $01^{100}$ & $5013.733 \pm 0.003$ & $-373.7 \pm 0.5$ & $-2.14 \pm 0.31$ \\
\hline $21^{1 d} 1$ & $01^{1 d} 0$ & & $-328.4 \pm 0.5$ & $-2.69 \pm 0.20$ \\
\hline $22^{2 c 1}$ & $02^{2 c 0}$ & $8+0.003$ & $-352.9 \pm 0.6$ & \\
\hline $22^{2 d_{1}}$ & $02^{2 a} 0$ & $5028.728 \pm 0.003$ & $-350.6 \pm 0.8$ & \\
\hline $06^{0} 1$ & $00^{\circ} 0$ & $5951.532 \pm 0.003$ & $-58.2 \pm 1.0$ & $10.77 \pm 0.67$ \\
\hline
\end{tabular}


Table III (Continued)

\begin{tabular}{|c|c|c|c|c|}
\hline \multicolumn{2}{|l|}{ Levels } & \multirow[t]{2}{*}{$v_{0}$} & \multirow[t]{2}{*}{$\left(B^{\prime}-B^{\prime \prime}\right) \times 10^{5}$} & \multirow[t]{2}{*}{$\left(D^{\prime}-D^{\prime \prime}\right) \times 10^{8}$} \\
\hline Upper & Lower & & & \\
\hline $\begin{array}{l}15^{1 c} 1 \\
15^{1 d} 1\end{array}$ & $\begin{array}{l}01^{1 c} 0 \\
01^{1 d} 0\end{array}$ & $6088.160 \pm 0.003$ & $\begin{array}{l}-328.6 \pm 0.5 \\
-235.7 \pm 0.6\end{array}$ & \\
\hline $14^{0} 1$ & $00^{9} 0$ & $6119.556 \pm 0.002$ & $-264.5 \pm 0.3$ & $4.63 \pm 0.09$ \\
\hline $22^{0} 1$ & $00^{\circ} 0$ & $6241.932 \pm 0.002$ & $-438.0 \pm 0.3$ & $-2.37 \pm 0.10$ \\
\hline $23^{1 c 1}$ & $01^{1 c 0}$ & & $-419.0 \pm 3.0$ & \\
\hline $23^{1 d} 1$ & $01^{1 d} 0$ & $6243.505 \pm 0.011$ & $-365.1 \pm 0.5$ & \\
\hline $30^{0} 1$ & $00^{\circ} 0$ & $6363.580 \pm 0.002$ & $-320.4 \pm 0.5$ & $-4.21 \pm 0.21$ \\
\hline $31^{1 c 1}$ & $01^{1 c 0}$ & & $-375.5 \pm 0.5$ & \\
\hline $31^{1 d} 1$ & $01^{1 d} 1$ & $6397.512 \pm 0.003$ & $-297.7 \pm 0.7$ & \\
\hline $01^{1}<3$ & $01^{1} c 0$ & & $-879.3 \pm 0.3$ & \\
\hline $01^{1 d 3}$ & $01^{1 d} 0$ & $6745.047 \pm 0.002$ & $884.9 \pm 0.3$ & \\
\hline $00^{03}$ & $00^{\circ} 0$ & $6780.140 \pm 0.002$ & $-887.2 \pm 0.2$ & \\
\hline $34^{0} 1$ & $00^{\circ} 0$ & $7481.367 \pm 0.003$ & $-489.3 \pm 1.1$ & $15.69+0.78$ \\
\hline $22^{0} 1$ & $00^{\circ} 0$ & $7599.935 \pm 0.002$ & $-431.8 \pm 0.4$ & \\
\hline $02^{03}$ & $00^{\circ} 0$ & $7981.175 \pm 0.002$ & $-799.1 \pm 0.3$ & \\
\hline $11^{1<3}$ & $01^{1 c} 0$ & & $-927.6 \pm 1.1$ & \\
\hline $11^{1 d 3}$ & $01^{1 d} 0$ & $8070.892 \pm 0.006$ & $-926.4 \pm 1.6$ & \\
\hline $10^{03}$ & $00^{\circ} 0$ & $8085.007 \pm 0.003$ & $-948.5+0.5$ & \\
\hline
\end{tabular}

a Arthur G. Maki, Earle K. Plyler, and Robert J. Thibault, J. Res. Nat. Bur. Stand., Sect. A, 67, 219 (1963).

b Ralph Oberly, K. Narahari Rao, Y. H. Hahn, and T. K. McCubbin, Jr., J. Mol. Spectrosc. 25, 138 (1968).

c Howard R. Gordon and T. K. McCubbin, Jr., J. Mol. Spectrosc. 19, 137 (1966). Note: The molecular constants for the data other than those pertaining to the above references have been obtained from R. E. Oberly, Ph. D. Dissertation, The Ohio State University (1970). In determining these constants, Oberly used the basic data given by Charles P. Courtoy, Ann. Soc. Sci. Bruxelles 73, 5-230 (1959).

from the Venus spectra (Connes et al., 1969) we have a fairly good set of data* available for the following isotopic varieties of carbon dioxide:

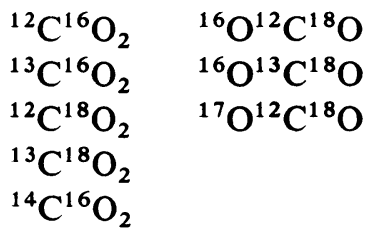

Using all this information, Cihla and Chedin (1971) determined the potential energy

* In view of the strong Fermi resonance between levels $v_{1}, v_{2}{ }^{l}, v_{3}$ and $\left(v_{1}-1\right),\left(v_{2}+2\right)^{l}, v_{3}$ theory requires that the designation of the levels cannot be made by past conventional procedures. The alternate solutions are somewhat complex to adopt and assimilate. Also, one quickly loses an intuitive appreciation of what bands we are referring to when we use the newer designations. This is an area which requires careful discussion and reasonable solutions. The designation of the levels given in Tables II-VIII has followed the notation used for the spectra of triatomic molecules. Many experimental results were reported in the literature employing these designations. Anyone interested in evaluating vibrational constants for $\mathrm{CO}_{2}$ should consult with the papers of Cihla and Chedin (1971) and Chedin and Cihla (1973). 
function for the carbon dioxide molecule. By extrapolating calculations to transitions involving higher $v$ values Chedin and Cihla (1973) predicted to good accuracy the emission lines involving levels $00^{\circ} 9,00^{\circ} 8, \ldots, 00^{\circ} 5$ measured subsequently. More experimental work is required to be done in the laboratory on the carbon dioxide

TABLE IV

Molecular constants (expressed in $\mathrm{cm}^{-1}$ ) of ${ }^{12} \mathrm{C}^{18} \mathrm{O}_{2}$ bands

\begin{tabular}{|c|c|c|c|c|}
\hline \multicolumn{2}{|l|}{ Levels } & & \multirow[t]{2}{*}{$\left(B^{\prime}-B^{\prime \prime}\right) \times 10^{5}$} & $\left(D^{\prime}-D^{\prime \prime}\right) \times 10^{8}$ \\
\hline Upper & Lower & & & \\
\hline $01^{1 c 1}$ & $01^{1 c} 0$ & & $-269.7 \pm 0.7$ & \\
\hline $01^{1 d} 1$ & $01^{1 d} 0$ & $2301.798 \pm 0.004$ & $-270.9 \pm 0.4$ & $35.2+8.5$ \\
\hline $00^{0} 1$ & $00^{\circ} 0$ & $2314.052 \pm 0.002$ & $-272.6 \pm 0.2$ & \\
\hline $04^{0} 1$ & $02^{\circ} 0$ & $3491.597 \pm 0.002$ & $-250.1 \pm 0.7$ & $3.1 \pm 0.5$ \\
\hline $03^{1 c 1}$ & $01^{110} 0$ & & $-287.8 \pm 0.2$ & $0.7 \pm 0.1$ \\
\hline $03^{1 d} 1$ & $01^{1 d} 0$ & $3493.894 \pm 0.001$ & $-270.5 \pm 0.3$ & $1.7 \pm 0.1$ \\
\hline $02^{01}$ & $00^{\circ} 0$ & $3525.205 \pm 0.001$ & $-295.9 \pm 0.1$ & $1.20 \pm 0.02$ \\
\hline $04^{2} 1$ & $02^{2} 0$ & $3549.388 \pm 0.002$ & $-293.7 \pm 1.2$ & $1.4 \pm 1.0$ \\
\hline $12^{01}$ & $02^{\circ} 0$ & $3602.934 \pm 0.002$ & $-258.6 \pm 0.6$ & $-2.9 \pm 0.4$ \\
\hline $10^{0} 1$ & $00^{\circ} 0$ & $3638.067 \pm 0.001$ & $-216.3 \pm 0.1$ & $-1.33 \pm 0.03$ \\
\hline $11^{1 c} 1$ & $01^{1 c} 0$ & & $-246.5 \pm 0.2$ & $-0.7 \pm 0.1$ \\
\hline $11^{1 d} 1$ & $01^{1 d} 0$ & $3646.043 \pm 0.001$ & $-216.8 \pm 0.2$ & $-1.0 \pm 0.1$ \\
\hline $20^{0} 1$ & $10^{\circ} 0$ & $3642.143 \pm 0.002$ & $-212.3 \pm 0.4$ & \\
\hline $12^{2} 1$ & $02^{2} 0$ & $3649.538 \pm 0.002$ & $-238.7 \pm 0.6$ & \\
\hline $05^{1 c} 1$ & $01^{1} c_{0}$. & & $-286.5 \pm 0.6$ & $2.1 \pm 0.3$ \\
\hline $05^{1 d} 1$ & $01^{1 d} 0$ & $674.256 \pm 0.002$ & $-247.5 \pm 0.5$ & $3.0 \pm 0.2$ \\
\hline $04^{0} 1$ & $00^{\circ} 0$ & $4721.920 \pm 0.001$ & $-280.0 \pm 0.2$ & $3.2+0.1$ \\
\hline $13^{1 c} 1$ & $01^{1} c 0$ & & $-295.8 \pm 0.2$ & \\
\hline $13^{1 d} 1$ & $01^{1 d} 0$ & $4827.253+0.002$ & $-254.0 \pm 0.2$ & \\
\hline $16^{0} 1$ & $00^{\circ} 0$ & $4833.262+0.001$ & $-288.3 \pm 0.3$ & $-2.0 \pm 0.1$ \\
\hline $20^{0} 1$ & $00^{\circ} 0$ & $4989.234+0.002$ & $-156.2 \pm 0.3$ & $-3.0+0.1$ \\
\hline $21^{1 c} 1$ & $01^{1} c_{0}$ & & $-213.0 \pm 0.3$ & \\
\hline $21^{1 d} 1$ & $01^{1 d} 0$ & $13 \pm$ & $-149.9+0.3$ & \\
\hline
\end{tabular}

R. Oberly, K. Narahari Rao, L. H. Jones, and M. Goldblatt, J. Mol. Spectrosc. 40, 356 (1971).

TABLE V

Molecular constants (expressed in $\mathrm{cm}^{-1}$ ) of ${ }^{13} \mathrm{C}^{18} \mathrm{O}_{2}$ bands

\begin{tabular}{|c|c|c|c|c|}
\hline \multicolumn{2}{|l|}{ Levels } & \multirow[t]{2}{*}{$v_{0}$} & \multirow[t]{2}{*}{$\left(B^{\prime}-B^{\prime \prime}\right) \times 10^{5}$} & \multirow[t]{2}{*}{$\left(D^{\prime}-D^{\prime \prime}\right) \times 10^{8}$} \\
\hline Upper & Lower & & & \\
\hline $01^{1 c} 1$ & $01^{1} 0^{a}$ & 2235.78 & -259 & \\
\hline $01^{1 d} 1$ & $01^{1 d} 0$ & & -262 & \\
\hline $00^{0} 1$ & $00^{\circ} 0^{a}$ & 2247.24 & -261 & \\
\hline $10^{0} 1$ & $00^{\circ} 0^{b}$ & $3545.029+0.002$ & -248.0 & $-1.6 \pm 0.2$ \\
\hline
\end{tabular}

a C. Chackerian, Jr. and D. F. Eggers, Jr., J. Mol. Spectrosc. 27, 59 (1968).

b R. Oberly, K. Narahari Rao, L. H. Jones, and M. Goldblatt, J. Mol. Spectrosc. 40, 356 (1971). 
TABLE VI

Molecular constants (expressed in $\mathrm{cm}^{-1}$ ) of ${ }^{12} \mathrm{C}^{16} \mathrm{O}^{18} \mathrm{O}$ bands

\begin{tabular}{|c|c|c|c|c|}
\hline \multicolumn{2}{|l|}{ Levels } & \multirow[t]{2}{*}{$v_{0}$} & \multirow[t]{2}{*}{$\left(B^{\prime}-B^{\prime \prime}\right) \times 10^{5}$} & \multirow[t]{2}{*}{$\left(D^{\prime}-D^{\prime \prime}\right) \times 10^{8}$} \\
\hline Upper & Lower & & & \\
\hline $03^{1} 0$ & $00^{0} 0^{\mathrm{a}}$ & 2049.68 & 48 & 1.0 \\
\hline $00^{01}$ & $00^{\circ} 0^{\mathrm{c}}$ & $2332.15 \pm 0.006$ & $-291.0 \pm 1.0$ & \\
\hline $20^{\circ} 0$ & $00^{\circ} 0^{\mathrm{a}}$ & 2500.73 & 25 & 0.0 \\
\hline $12^{00}$ & $00^{0} 0^{\mathrm{a}}$ & 2614.20 & -43 & 0.0 \\
\hline $04^{0} 0$ & $00^{\circ} 0^{\mathrm{a}}$ & 2757.14 & 90 & 0.0 \\
\hline $02^{01}$ & $00^{\circ} 0^{d}$ & $3571.140 \pm 0.001$ & $-287.8 \pm 0.2$ & $1.7 \pm 0.1$ \\
\hline $10^{01}$ & $00^{\circ} 0^{d}$ & $3675.139 \pm 0.001$ & $-262.3 \pm 0.3$ & $-2.2 \pm 0.1$ \\
\hline $00^{02}$ & $00^{\circ} 0^{b}$ & $4639.530 \pm 0.003$ & $-590.2 \pm 0.8$ & 0 \\
\hline $02^{02}$ & $00^{0} 0^{\mathrm{e}}$ & 5858.022 & -567.4 & -1.47 \\
\hline
\end{tabular}

a C. V. Berney and D. F. Eggers, Jr., J. Chem. Phys. 40, 990 (1964).

b F. A. Diaz, J. Opt. Soc. Am. 53, 203 (1963).

c R. Oberly, K. Narahari Rao, Y. H. Hahn, and T. K. McCubbin, Jr., J. Mol. Spectrosc. 25, 138 (1968).

d R. Oberly, K. Narahari Rao, L. H. Jones, and M. Goldblatt, J. Mol. Spectrosc. 40, 356 (1971).

e L. G. Young, Icarus 13, 270 (1970).

TABLE VII

Molecular constants (expressed in $\mathrm{cm}^{-1}$ ) of ${ }^{13} \mathrm{C}^{16} \mathrm{O}^{18} \mathrm{O}$ bands

\begin{tabular}{|c|c|c|c|c|}
\hline Levels & & $v_{0}$ & $\left(B^{\prime}-B^{\prime \prime}\right) \times 10^{5}$ & $\left(D^{\prime}-D^{\prime \prime}\right) \times 10^{8}$ \\
\hline Upper & Lower & & & \\
\hline $\begin{array}{l}00^{01} \\
00^{02}\end{array}$ & $\begin{array}{l}00^{0} 0^{\mathrm{a}} \\
00^{0} 0^{\mathrm{b}}\end{array}$ & $2265.973 \pm 0.010$ & $-275.8 \pm 0.7$ & \\
\hline 0002 & $00^{\circ} 0^{\mathrm{b}}$ & 4508.748 & -557.82 & 0 \\
\hline
\end{tabular}

a R. Oberly, K. Narahari Rao, Y. H. Hahn, and T. K. McCubbin, Jr., J. Mol. Spectrosc. 25, 138 (1968).

b L. G. Young, Icarus 11, $66(1969)$.

TABLE VIII

Molecular constants (expressed in $\mathrm{cm}^{-1}$ ) of ${ }^{12} \mathrm{C}^{17} \mathrm{O}^{18} \mathrm{O}$ bands

\begin{tabular}{lllll}
\hline Levels & & $v_{0}$ & $\left(B^{\prime}-B^{\prime \prime}\right) \times 10^{5}$ & $\left(D^{\prime}-D^{\prime \prime}\right) \times 10^{8}$ \\
\cline { 1 - 2 } & Lower & & \\
\hline $10^{0} 1$ & $00^{0} 0^{\mathrm{a}}$ & $3655.130 \pm 0.002$ & $-238.1 \pm 0.8$ & $-2.7 \pm 0.6$
\end{tabular}

a R. Oberly, K. Narahari Rao, L. H. Jones, and M. Goldblatt, J. Mol. Spectrosc. 40, 356(1971). 
TABLE IX

Molecular constants for the ground states of the carbon dioxide molecule and its isotopic varieties

\begin{tabular}{|c|c|c|c|}
\hline $\begin{array}{l}\text { Isotopic } \\
\text { species }\end{array}$ & $B_{00} 0_{0}\left(\mathrm{~cm}^{-1}\right)$ & $D_{00^{0}}\left(\mathrm{~cm}^{-1}\right)$ & Reference \\
\hline${ }^{12} \mathrm{C}^{16} \mathrm{O}_{2}$ & $0.3902191 \pm 0.0000032$ & $(13.31 \pm 0.12) \times 10^{-8}$ & 1 \\
\hline${ }^{12} \mathrm{C}^{16} \mathrm{O}^{18} \mathrm{O}$ & $0.36818 \pm 0.00005$ & $(11.6 \pm 5.0) \times 10^{-8}$ & 2 \\
\hline${ }^{12} \mathrm{C}^{17} \mathrm{O}^{18} \mathrm{O}$ & $0.35702 \pm 0.00005$ & $(14.7 \pm 2.7) \times 10^{-8}$ & 2 \\
\hline${ }^{12} \mathrm{C}^{18} \mathrm{O}_{2}$ & $0.346799 \pm 0.00013$ & $(10.4 \pm 2) \quad \times 10^{-8}$ & 2 \\
\hline${ }^{13} \mathrm{C}^{16} \mathrm{O}_{2}$ & 0.39027 & $\times 10^{-8}$ & 3 \\
\hline${ }^{13} \mathrm{C}^{18} \mathrm{O}_{2}$ & 0.34679 & $(9.3 \pm 1.9) \times 10^{-8}$ & 2 \\
\hline${ }^{13} \mathrm{C}^{16} \mathrm{O}^{18} \mathrm{O}$ & 0.36812 & & 3 \\
\hline${ }^{14} \mathrm{C}^{16} \mathrm{O}_{2}$ & 0.39021 & $\times 10^{-8}$ & 4 \\
\hline
\end{tabular}

1. T. K. McCubbin, Jr., Josef Pliva, Robert Pulfry, William Telfair, and Terry Todd, 'The Emission Spectrum of ${ }^{12} \mathrm{C}^{16} \mathrm{O}_{2}$ from 4.2 to 4.7 Microns', J. Mol. Spectrosc. 49, 136(1974). The authors give aslo $H_{00} 0_{0}=(-1.26 \pm 1.33) \times 10^{-13} \mathrm{~cm}^{-1}$.

2. R. Oberly, K. Narahari Rao, L. H. Jones, and M. Goldblatt, J. Mol. Spectrosc. 40, 356 (1971). 3. R. E. Oberly, Ph. D. Dissertation, The Ohio State University (1970). The basic data given in Reference 4 have been used to recompute the constants.

4. Charles P. Courtoy, Infrared spectrum at high dispersion and molecular constants of $\mathrm{CO}_{2}, \mathrm{Ann}$. Soc. Sci. Bruxelles 73, 5-230 (1959). (In French.) [G. R. Wilkinson values, unpublished, are quoted on p. 150.]

molecule and its isotopic varieties including measurements at high temperatures, long paths, and high pressures.

\section{6. $\mathrm{NO}_{2}, \mathrm{HNO}_{3}$ and $\mathrm{NO}$}

The interest in the infrared spectra of $\mathrm{NO}_{2}$ and $\mathrm{HNO}_{3}$ have been prompted as a result of the identification of these molecular species in the Earth's stratosphere (Goldman et al., 1970; Ackerman and Muller, 1972; Murcray et al., 1968, 1969). The infrared spectra of $\mathrm{NO}_{2}$ are complex and their interpretation has progressed significantly during the past year (Hurlock et al., 1973a, b). The question of the occurrence and abundance of NO in the stratosphere is being vigorously pursued at the present time and references to two most recent articles (Girard et al., 1973; Ridley et al., 1973) are given to illustrate the type of experiments being undertaken with balloon borne samplers and by measurements made during flights of a supersonic transport. In addition, more recently a letter on detection of nitric oxide in the lower atmosphere has been published (Toth et al., 1973). Needless to mention that the laboratory data for the spectral positions and intensities of some of the infrared bands used in these pursuits are very significant undertakings.

\section{A Few Comments on Intensity Studies in Infrared Spectra}

It should be emphasized that even if we are interested in intensities, it is important not to lose sight of the value of having accurate spectral positions because they are vitally needed for the assignments of the bands studies. This includes the assignments 
of the rotational structure observed in these bands recorded under high resolution. Such assignments assure the identifications of the bands to specific molecular species.

At the present time, excellent intensity work is being conducted in different laboratories employing mainly medium resolution infrared spectrographs. In the future, higher resolution instruments will be used and should provide valuable information.

An examination of the published literature reveals avoidable confusion especially in connection with the nomenclature adopted by spectroscopists reporting intensity data. The following sample shows that there exist numerous preferences for the units in which the strengths of the lines are being reported:

\section{Strengths of the lines, $S$}

$$
\begin{aligned}
& \mathrm{cm}^{-1}(\mathrm{~cm} \mathrm{~atm})^{-1} \\
& \mathrm{~cm}^{-1}(\mathrm{~cm} \mathrm{~atm})^{-1} \mathrm{STP} \\
& \mathrm{cm}^{-1}(\mathrm{~m} \mathrm{~atm})^{-1} \\
& \mathrm{~cm}^{-1}(\mathrm{~km} \mathrm{~atm})^{-1} \\
& \mathrm{~cm}^{-1}\left(\mathrm{~km} \mathrm{amagat}^{-1}\right. \\
& \mathrm{cm}^{-1}\left(\frac{\text { molecules }}{\mathrm{cm}^{2}}\right)^{-1} \text { or } \mathrm{cm}^{-1}\left(\frac{\text { molecules }}{\mathrm{cm}^{3}} \times \mathrm{cm}\right)^{-1} \\
& \mathrm{~cm}^{-1}\left(\frac{\text { grams }^{-1}}{\mathrm{~cm}^{2}}\right)^{-1} \text { or } \mathrm{cm}^{-1}\left(\frac{\text { grams }}{\mathrm{cm}^{3}} \times \mathrm{cm}\right)^{-1}
\end{aligned}
$$

Wave numbers per molecule per $\mathrm{cm}^{2}$ at $295^{\circ} \mathrm{C}$

The last usage in the above table is an unfortunate consequence of a trend to use the word 'wave number' as synonymous to $\mathrm{cm}^{-1}$. The word 'wave number' means reciprocal of the word 'wavelength' and the units for it depend upon the units used for wavelength. Commission 14 of the IAU has done yeoman service in the past in providing leadership with respect to wavelength standards. Perhaps they can consider the various aspects of spectroscopic nomenclature and units, in general, so that all scientists interested in the field may communicate with each other without creating sources for errors.

\section{Acknowledgements}

I would like to express my gratitude to Dr Tobias Owen for presenting this paper on my behalf at the Copernicus Symposium IV held in Torun, Poland, during September $5-8,1973$. Some of the problems discussed here have been the result of an examination of the problems of interest to the Department of Transportation, Climatic Impact Assessment Program. Finally, my thanks are also due to Dr Lawrence Weller for his help in preparing the manuscript.

Note added in proof. In a very recent communication, S. T. Ridgway (Astrophys. $J$. 187 (1974), L41), reported the identification of ethane $\left(\mathrm{C}_{2} \mathrm{H}_{6}\right)$ and acetylene $\left(\mathrm{C}_{2} \mathrm{H}_{2}\right)$ in the atmosphere of Jupiter. This was done by using a Fourier transform spectrometer at a resolution of $1.3 \mathrm{~cm}^{-1}$ in the range $750-1300 \mathrm{~cm}^{-1}(10$ micron window $)$. 
This identification points to important possibilities for the future. In fact, Ridgway concluded:

The spectra described here were obtained in $3 \mathrm{~h}$ with a prototype instrument. Detectors 10 times more sensitive and telescopes of 3 times greater aperture are available. Within a few years much higher spectral resolution in the 10- $\mu$ window will be available. The ethane and acetylene emission features will be useful for future studies of the temperature inversion. We wish to emphazise that at this time the analysis is limited by the scarcity of molecular data, especially line intensities, for relatively simple organic molecules.

\section{References}

Ackerman, M. and Muller, C.: 1972, Nature 240, 300.

Cihla, Z. and Chedin, A.: 1971, J. Mol. Spectrosc. 40, 337.

Chedin, A. and Cihla, Z.: 1973, J.Mol.Spectrosc. 47, 554.

Connes, P., Connes, J., Benedict, W. S., and Kaplan, L. D.: 1967, Astrophys. J. 147, 1230.

Connes, P., Connes, J., Kaplan, L. D., and Benedict, W. S.: 1968, Astrophys. J. 152, 731.

Connes, J., Connes, P., and Maillard, J.: 1969, Near Infrared Spectra of Venus, Mars, Jupiter and Saturn Atlas, Vol. 1, C.N.R.S., Paris.

Cowan, M. and Gordy, W.: 1957, Bull. Am. Phys. Soc. 2, 212.

Dunham, J. L.: 1932, Phys. Rev. 41, 721.

Fleming, H. E. and Rao, K. Narahari: 1972, J. Mol.Spectrosc. 44, 189.

Girard, A., Fontanella, J. C., and Gramont, L.: 1973, Compt. Rend. Acad. Sci. Paris 276, Ser. B, 845.

Goldman, A., Murcray, D. G., Murcray, F. H., Williams, W. J., and Bonomo, F. S.: 1970, Nature 225, 443.

Hall, D. N. B.: 1973, Astrophys. J. 182, 977.

Helminger, P., DeLucia, F. C., and Gordy, W.: 1970, Phys. Rev. Letters 25, 1397.

Herzberg, G. and Rao, K. Narahari : 1949, J. Chem. Phys. 17, 1099.

Hurlock, S. C., Rao, K. Narahari, Weller, L. A., and Yin, P. K. L.: 1973a, J. Mol. Spectrosc. 48, 372.

Hurlock, S. C., Lafferty, W. J., and Rao, K. Narahari: 1974, J. Mol. Spectrosc. 50, 246.

Kaplan, L. D., Connes, J., and Connes, P.: 1969, Astrophys. J. 157, L187.

Kirschner, S. M. and Watson, J. K. G.: 1973, J. Mol. Spectrosc. 47, 234.

Locke, J. L. and Herzberg, L.: 1953, Can. J. Phys. 31, 504.

Mantz, A. W., Nichols, E. R. Alpert, B. D., and Rao, K. Narahari: 1970, J. Mol. Spectrosc. 35, 325.

Mantz, A. W., Watson, J. K. G., Rao, K. Narahari, Albritton, D. L., Schmeltekopf, A. L., and Zare, R. N.: 1971, J. Mol. Spectrosc. 39, 180.

Migeotte, M.: 1959, Phys. Rev. 75, 1108.

Migeotte, M. and Neven, L.: 1950, Physica 16, 423.

Murcray, D. G., Kyle, T. G., Murcray, F. H., and Williams, W. J.: 1968, Nature 218, 78.

Murcray, D. G., Kyle, T. G., Murcray, F. H., and Williams, W. J.: 1969, J. Opt. Soc. Am. 59, 1131.

Rank, D. H., Guenther, A. H., Saksena, G. D., Shearer, J. N., and Wiggins, T. A.: 1957, J. Opt . Soc. Am. 47, 686.

Rank, D. H., Pierre, A. G. St., and Wiggins, T. A.: 1965, J. Mol. Spectrosc. 18, 418.

Rao, K. Narahari: 1950, J. Chem. Phys. 18, 213.

Rao, K. Narahari, Humphreys, C. J., and Rank, D. H.: 1966, Wavelengths Standards in the Infrared, Academic Press, New York.

Rao, K. Narahari and Mathews, C. W. (eds.): 1972, Molecular Spectroscopy: Modern Research, Academic Press, New York, Ch. 7.

Ridley, B. A., Schiff, H. I., Shaw, A. W., Bates, L., Howlett, C., LeVaux, H., Megill, L. R., and Aschenfelter, T. E.: 1973, Nature, 245, 310.

Roh, W. B. and Rao, K. Narahari: 1974, J. Mol. Spectrosc. 49, 317.

Rosenbloom, B., Nethercot, A. H., Jr., and Townes, C. H.: 1958, Phys. Rev. 109, 400.

Toth, R. A., Farmer, C. B., Schindler, R. A., Raper, O. F., and Schaper, P. W.: 1973, Nature 244, 7.

Weinberg, J. M., Fishburne, E. S., and Rao, K. Narahari: 1965, J. Mol. Spectrosc. 18, 428.

Wilson, R. W., Jefferts, K. B., and Penzias, A. A.: 1970, Astrophys. J. 161, L43. 


\section{DISCUSSION}

Feigelson: What are the upper limits of pressure and temperature for which we have good laboratory spectroscopic data for $\mathrm{CO}_{2}$ ? We have not such data for real Venus conditions.

Owen and Young: It has not yet been possible to match the spectral resolution achieved by Dr Connes in Venus spectra with long path lengths in the laboratory in the region $1.2 \mu$. At shorter wavelengths Gerhard Herzberg has published results in the early 1950's, and D. Rank in mid-1960's. Herzberg's absorption tube was capable of measuring a $1 \mathrm{~km}$ path of gas at pressures of 1-2 atm. Individual rotational lines were resolved, but at a resolution somewhat less than that which could be obtained for Venus. However Herzberg's absorption tube could not be heated or cooled, nor could it sustain pressures much larger than atmospheric pressure. Long paths at high pressure and temperature have also not received study in this region of the spectrum. 